\title{
ASCULTATION OF THE CHEST
}

DR. STEPHEN C. MORRISON,* M.A., M.B., B.Chir., M.R.C.P.

\section{SUMMARY}

The stethoscope and its use are described. Aiçurrent classification of breath sounds, voice sounds and adventitious sounds is presented, and its use in diagnosis outlined. Finally, the value of auscultation to the physiotherapist is discussed.

\section{INTRODUCTION}

The invention of the stethoscope by Laennec in 1818 was a major advance in medicine; the instrument allowed diagnosis and assessment of cardio-respiratory disorders to be carried out with precision not previously attainable, and to this day the stethoscope remains an indispensable part of the physician's armamentarium.

For physiotherapists, however, the situation is not so clear cut. A substantial proportion of physicians and surgeons view with deepest suspicion the sight of a physiotherapist auscultating a patient's chest. In the eyes of these critics it verges on blasphemy for the physiotherapist to own a stethoscope, or to carry one about as a matter of routine. The opposite viewpoint is held by many members of the physiotherapy profession, believing auscultation to be very much their business, and a valuable source of information pertinent to the performance of their work. I do not propose to enter into the pros and cons of this controversy. I consider it beyond dispute, however, that if the stethoscope is to be used by physiotherapists, it should be used correctly, with insight and understanding. This article is written to help achieve this end.

\section{THE STETHOSCOPE}

(Greek stethos, the chest; skopeein, to explore).

There are three components of the modern stethoscope. They are the chest piece, the tubing and the binaural.

The chest piece may be of the bell type, diaphragm type, or a combination of these two. The two are really variants of the same principle - that of a damped diaphragm system. The area of skin enclosed by the bell behave as a diaphragm, the tautness of which may be varied by the pressure applied. The firmly applied

* Respiratory Clinic, Groote Schuur Hospital, Cape Town.

Received 16 July 1980.

\section{OPSOMMING}

Die stetoskoop en die gebruik daarvan word beskryf. 'n Huidige klassifikasie van asemhalingsklanke, stemhebbende klanke en bykomende klanke word voorgestel, en die gebruik daarvan in diagnostiek word omskryf. Ten slotte word die waarde van ouskultasie vir die fisioterapeut bespreek.

bell therefore subtends an area of taut skin which behaves in a similar way to the diaphragm chest piece; it filters out low frequency sounds, allowing the higher frequencies to come through. It should be remembered, though, that the volume of sound is related to the area of the chest piece, so that the diaphragm chest piece, being larger, will in general produce a higher amplitude of sound than the firmly applied bell. In contrast, the softly applied bell subtends an area of lax skin which will have a much lower resonant frequency, favouring the transmission of low frequency sounds.

Heart sounds, and some cardiac murmurs, are in the lower frequency range $(20-115$ cycles/sec), so that cardiologists will usually prefer the softly applied (1) when auscultating the heart.

Lung sounds, however, and especially abnormal lung sounds, are in the higher frequency range $(200-2000$ cycles/sec), so that use of the diaphragm is generally preferable, although the firmly applied bell could be used. Advantages of the diaphragm include the higher amplitude of sound, as already mentioned, and the easier application over an uneven or bony chest cage, where incomplete contact between skin and the rim of the bell would result in complete loss of sound. The firmly applied bell may be useful on occasion; for example, in confirming the presence of fine adventitious sounds which can sometimes be generated artificially by movement of the diaphragm on the skin surface. The bell is also useful in children, for whom the diaphragm may be inappropriately large, although paediatric stethoscopes are available.

The tubing is of considerable importance in the efficiency of a stethoscope. Sound loss can result from the use of incorrect dimensions or materials. The material should be firm, inert, reasonably thick and polished in its internal bore for maximum transmission. Loss of high frequencies can result if the volume of the system is too large or if the diameter of the tubing is too fine. A good. compromise is Tygon tubing, as used in the 
Littman stethoscope (Littman, 1961), with a lumen $3 \mathrm{~mm}$ in diameter and $35 \mathrm{~cm}$ in length from chest piece to the base of the " $Y$ " section. Longer tubes add nothing in convenience, and seriously impair sound conduction. Some physicians prefer a twin-tubed instrument, rightly maintaining that loss of sound occurs at the "Y" section in single-tubed stethoscopes. This benefit of the twin-tubed instrument is partly offset by the necessarily larger volume of the system. Furthermore, twin tubes exhibit an annoying tendency to rub together, producing extraneous sounds. Hence, the more compact single-tubed instrument is preferable for general use.

The binaural is the final link in the chain between chest and ear, and is the source of much difficulty with auscultation. The ear piece should be as large as possible; no smaller than $12 \mathrm{~mm}$ and as large as $15 \mathrm{~mm}$. They are intended to occlude the external auditory canals, not to invade them. For most individuals, the external auditory canal is directed about $20^{\circ}$ anteriorly from its meatus, and the binaural should be adjusted ntil a comfortable fit is achieved. A firm spring tension 5 required to hold the binaural in place, and this will not be uncomfortable provided the earpieces are large enough. Leakage and loss of sound will result if spring tension is inadequate.

Prevention of leaks is of great importance if the stethoscope is to be used efficiently. The chief sources of leaks are the earpieces, the bell-diaphragm changeover mechanism, and cracks in the diaphragm or tubing. A useful test of the acoustic seal of the whole system is withdrawal of the chest piece quickly from the precordium, when a painful sensation should be produced at the ear.

\section{LUNG SOUNDS}

In the most important discussion of lung sounds since Laennec, Forgacs (1978) has placed their genesis and classification on a scientific and practical footing. The terms he enumerates are unambiguous, and in general make no implications $a b r^{*}$ It the source of sounds, except where this has been clearly elucidated. These terms have been widely adopted, and will be used throughout this article. Older terms should now be abandoned. The classification is summarised in Figure 1.

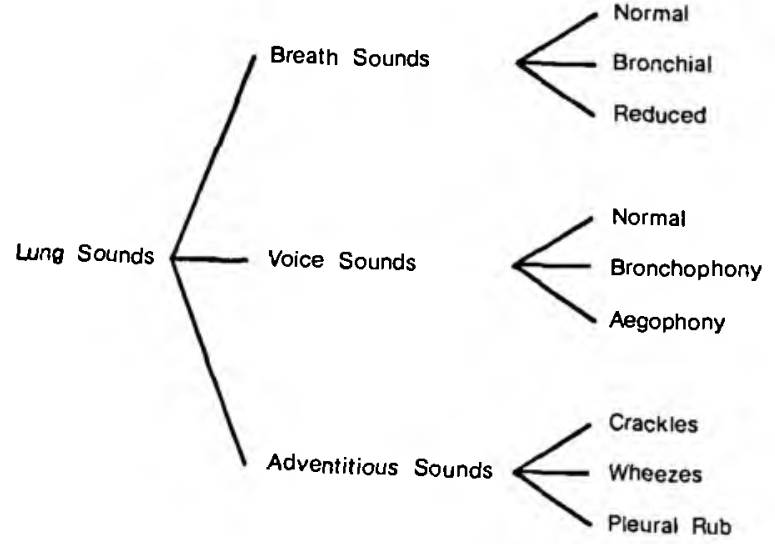

Fig. 1

\author{
Breath Sounds \\ Normal Breath Sounds
}

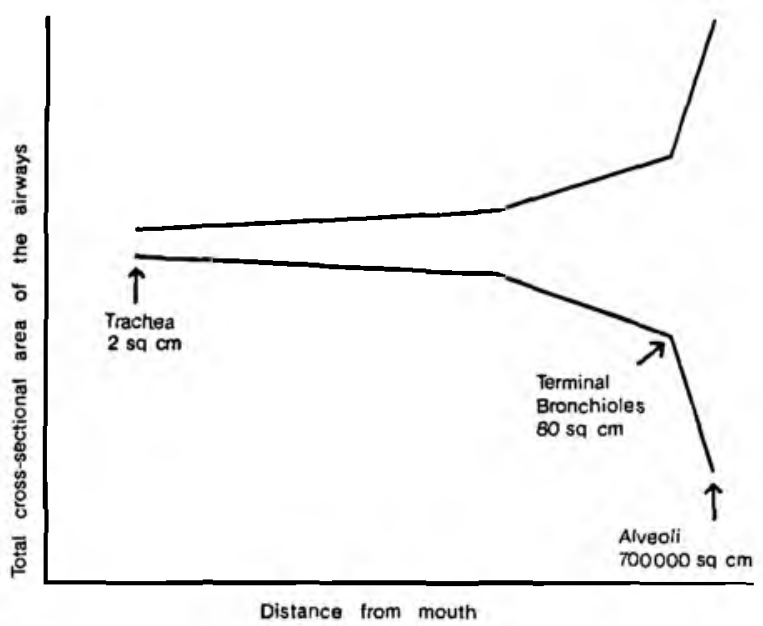

Fig. 2

The total cross-sectional area of the airways becomes progressively larger from trachea to alveoli (Figure 2). Hence, if total air flow in the central and peripheral airways is to be the same (as it must be), the velocity of the airstream will be considerably higher in the central airways. Thus flow in the central airways will be more prone to turbulence, and hence the generation of sound. The source of normal breath sounds is now known to be these central airways, i.e. the trachea and larger bronchi.

A stethoscope placed over the trachea will reveal breath sounds comprising frequencies from 200 to 2000 cycles/sec, and audible throughout most of the ventilatory cycle. In contrast, a stethoscope placed on the chest wall will demonstrate the sound absorptive and acoustic filtering effects of the lung tissue situated between the large bronchi and the chest wall. The sound amplitude is much lower owing to absorption by the lung; the frequency range is also lower, (from 200 to 600 cycles/ $\mathrm{sec})$, because lung tissue behaves as a "low pass" filter which selectively absorbs the higher frequencies. A consequence of the lower amplitude is that breath sounds are only audible when the flow rate is above about 0,75 litres/sec. This still includes most of inspiration, but only about one third of the longer expiratory phase (Figure 3). Thus normal breath sounds are low frequency, low amplitude sounds, heard during inspiration and the first third of expiration.

\section{Bronchial breath sounds or bronchial breathing}

If the alveoli become filled with fluid rather than air, there is relatively little loss of sound by absorption or filtration. The breath sounds heard at the chest will will therefore resemble those heard over the trachea, i.e. they will be greater in amplitude, higher in frequency, and audible over a longer proportion of the ventilatory cycle. This is called bronchial breathing, and is characteristic of the sound heard over an area of consolidation, e.g. in lobar pneumonia.

In certain individuals, and particularly in children, normal breath sounds may take on a slightly bronchial 


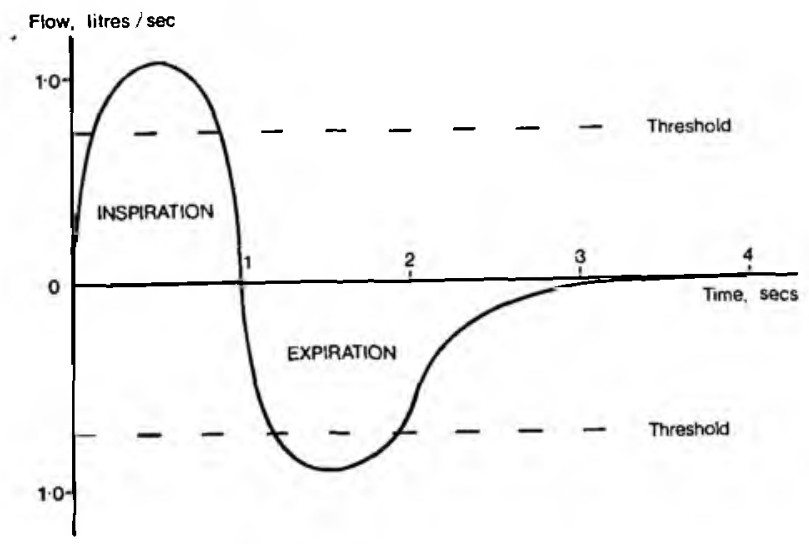

Fig. 3

quality owing to the proximity of the trachea and chest wall. This occurs especially in the central inter-scapular area on the back, and is of little consequence provided it is remembered.

A variant of bronchial breathing is amphoric breathing where low frequency breath sounds appear selectively amplified, and the bronchial breathing has a hollow ring to it. This type of breath sound is not infrequently heard over a large cavity.

\section{Reduced or absent breath sounds}

Breath sounds may be reduced or absent where the lung is separated from the chest wall, either by air (as in pneumothorax) or by fluid (as in pleural effusion). The two conditions are generally distinguishable on other grounds, such as percussion note. Reduced breath sounds are also a feature of emphysema, but will usually be accompanied by adventitious sounds in this condition. Localised reduction in breath sounds may imply a totally obstructed lobar or segmental bronchus.

\section{Voice Sounds}

\section{Normal Voice Sounds}

In the same way that breath sounds are attenuated and filtered by air-filled lung tissue, so the sounds of phonated and whispered speech, which originate in the larynx and mouth, are likewise attenuated and filtered by the lung. Speech is therefore reduced to a low frequency mumble in which words cannot be distinguished, and whispering is not normally audible at the chest wall.

\section{Bronchophony}

Where the lung between the central airways and the chest wall becomes fluid-filled as in consolidation, the higher frequency voice sounds are transmitted and words become distinguishable. This is known as bronchophony. Whispered speech, which is composed entirely of higher frequency sounds, becomes clearly transmitted to the chest wall, a sign known as whispering pectoriloquy.
These three signs (bronchial breathing, bronchophony and whispering pectoriloquy) form the triad of auscultatory signs of consolidation and all are the result of the same acoustic phenomenon.

\section{Acgophony}

At the upper border of a plcural effusion, where visceral and parietal pleura interface with pleural fluid, there is a tendency for lower frequency sounds to be selectively attenuated. Voice sounds are therefore transmitted with a high-pitched, nasal or bleating quality, and this is known as aegophony.

\section{Arlventitious Sounds}

\section{Crackles}

Crackles are short, explosive, non-musical sounds. Their genesis has been a subject of considerable confusion for many years. The most important single fif to remember is that, except in the case of the very. largest airways, the resistance offered by surface tension and viscosity of bronchial secretions is too high to allow "bubbling" of air through secretions. For practical purposes, we should try to assign crack'es into one of three different subtypes as this differentiation may be made with relative ease on acoustic grounds. While some inferences may be drawn in regard to underlying pathology, such inferences should be made with caution, and almost never from auscultatory evidence alone.

\section{Fine, late respiratory crackles}

If an alveolus of the lung becomes closed during any part of the breathing cycle, then during the next inspiration, a pressure gradient will build up trying to re-open the closed alveolus. There will come a point where the inspiratory force is strong enough to overcome the combined surface tension and elastic forces; at that point the alveolus will open abruptly, with rapid movement of air, thus equalising the air pressure between the alveolus and its conducting airway. This rapid equalisation of pressure is accompanied by a fine, high-pitched crackle, usually occurring late in inspiration. Crackles of this type are therefore heard in situations characterised by alveolar closure during some part of the ventilatory cycle. Such closure does not occur in tidal breathing in healthy young adults, but it may do so if Functiol/1, Residual Capacity (FRC)* falls, or if pulmonary elast recbil increases, either of these situations tending to bring alveoli nearer to collapse. Examples of the former are the atelectasis which follows abdominal surgery, and states of muscular weakness such as myasthenia gravis. Examples of increased elastic recoil would include fibrosing alveolitis, pneumoconioses (e.g. asbestosis) and interstitial pulmonary oedema. It is worth repeating that the crackles which occur in the latter condition derive from the effect of interstitial fluid on elastic recoil of the lung, and not from the presence of free fluid in the alveoli. In all these conditions, the alveoli nearest the closure will be those in the lowest part of the lung, being subject to the compressive weight of the overlying lang tissue. This fact may be demonstrated, for example in a patient with fibrosing alveolitis, by observation of the effect of posture (head down, supine, prone, standing etc.) when the crackles will localise cach time to the lowest zone. In summary, fine, lateinspiratory crackles are heard in interstitial pulmonary disorders or in otherwise normal lungs when FRC is

* volume of air remaining in the lungs at the end of a normal breath. 
reduced. Not surprisingly, these crackles are unaffected by coughing, and do not occur on expiration.

\section{Early inspiratory and expiratory cracklcs}

This kind of sound is commonly heard in patients with chronic airflow obstruction. The crackles arise from large airways, are low pitched and rather scanty. They are thought to be due to intermittent opening of lightly closed bronchi, and each crackle corresponds to the passage of a single bolus of gas through the closed airway. The tendency for these airways to close may be related to structural changes known to occur in chronic airflow obstruction, but this is incompletely understood at the present time. Nevertheless, crackles of this type are among the commonest encountered in the practice of respiratory medicine. They occur both on inspiration and on expiration, and are unaffected by coughing or posture.

\section{S} arse crackles related to bronchial secretions

Despite the foregoing, there remains a group of patients in whom unexpectorated secretions lie in the trachea and larger bronchi. The movement of air over and through these secretions gives rise to crackles of the loudest and coarsest type, both on inspiration and on expiration. These crackles are markedly reduced after coughing or endotracheal suction. Their presence correlates with inability or unwillingness to cough rather than with a specific disease and they occur in chronic bronchitis, bronchiectasis, pneumonia, tuberculosis, florid pulmonary oedema, or any condition giving rise to large volumes of intra-bronchial fluid. Often these patients will be in a moribund state, and this is the situation aptly portrayed in the lay term "death rattle".

\section{Wheeze}

Wheezes are musical sounds emanating from the lungs. They are heard on auscultation of the chest, but may also be audible without a stethoscope. Although the precise mechanisms of wheeze production are complex, the principle is that air flowing through narrowed bronchi sets up vibrations in the walls of those bronchi. Wheeze therefore occurs in conditions where airways are obstructed, e.g. asthma and chronic bronchitis. Only in large and medium-sized bronchi is there sufficient air flow to generate wheeze, and the common belief that -igh-pitched wheezes originate from the smallest periseral airways is probably untrue. The pitch of a wheeze cepends more on the resonant frequency of the bronchial wall than the dimensions of the bronchus. Because expiration is associated with compression and further narrowing of intrathoracic airways, wheeze is usually more prominent during expiration; indeed a wheeze present only on inspiration strongly implies the presence of an extra-thoracic obstruction, e.g. in the larynx; such inspiratory wheeze may be accompanied by stridor, a loud phonated sound occurring during inspiration when the larynx is obstructed.

Two notes of caution require mention in the context of wheeze. One is that wheeze does not always imply disease of obstructive type. In pulmonary oedema, for example, where alveolar closure occurs during the breathing cycle, alveoli on the point of closure may be a source of wheeze, and this should not be taken to indicate the presence of asthma as well. The second point to beware of is that severe airflow obstruction may be present in the complete absence of wheeze. In status asthmaticus, for example, obstruction may be so severe that there is just not enough airflow to generate wheeze.

\section{Pleural friction rub}

The third category of adventitious sounds applies to conditions where parietal or visceral pleural layers, or both, become inflamed. The two layers then generate sounds as they rub together during breathing. The sounds are typically high-pitched, and of a slightly "rough" character; they may occasionally be squeaky, reminiscent of brand-new leather shoes. The cadence of sound heard during inspiration is typically reversed during expiration. Pleural friction rub is most commonly observed in pleurisy, pneumonia and pulmonary infarction (such as follows pulmonary embolism).

\section{PRACTICAL USES OF AUSCULATION}

Auscultation is a valuable aid to diagnosis and follow-up. Auscultatory findings should, however, always be used in conjunction with full physical examination, with the clinical history and, where possible, with the chest radiograph. A willingness to auscultate without recognising these other factors is both inept and potentially dangerous. Examples have appeared above where auscultatory findings alone would have been ambiguous, or even frankly misleading.

Nevertheless, having accepted these constraints, the physiotherapist may be able to derive much useful information via the stethoscope, and so be able to tailor therapy for the patient's maximum benefit.

Questions frequently asked concern the quality of ventilation to the various lung regions. Is there mucus plugging in a certain area? Or an inhaled foreign body? Are the lung bases atelectatic? Is pneumonia developing? Or pneumothorax? Which segments or lobes should be posturally drained? These are all examples of questions to which auscultation may provide a part of the answer. Other questions relate to the general adequacy of ventilatory function. Is the patient retaining secretions despite attempts to cough? Could he be treated adequately if the endotracheal tube were removed? What effect are inhaled bronchodilators having?

\section{CONCLUSION}

It should by now be apparent that auscultation is by no means a simple procedure. Acquisition of skill demands constant practice. Used correctly and with circumspection, however, the technique can provide valuable information to the physiotherapist engaged in the treatment and prevention of chest disease.

\section{References}

Forgacs, P. (1978): Lungs sounds. Bailliere Tindall. Lotidon.

Littman, D. (1961): An Approach to the ideal stethoscope. J. Amer. Med. Ass., 13, $504-505$. 